\title{
Design, Synthesis, and Docking Studies of Arylhydrazone Compounds and Evaluation of Their Platelet Aggregation Inhibitory Effect and Cytotoxicity
}

\section{Maryam H. Klidsar ( $\square$ maryamheydarpour3@gmail.com )}

Universidade de Lisboa Faculdade de Farmácia: Universidade de Lisboa Faculdade de Farmacia https://orcid.org/0000-0003-4836-3719

\section{Marjan Esfahanizadeh}

Shahid Beheshti University of Medical Sciences School of Pharmacy

\section{Pantea Haghverdi}

Shahid Beheshti University of Medical Sciences School of Pharmacy

\section{Salimeh Amidi}

Shahid Beheshti University of Medical Sciences School of Pharmacy

\section{Farzad Kobarfard}

Shahid Beheshti University of Medical Sciences School of Pharmacy

\section{Research Article}

Keywords: antiplatelet, platelet aggregation, $\mathrm{N}$-isonicotinohydrazide, arylhydrazone, Synthesis, Docking, Cytotoxicity test

Posted Date: January 11th, 2022

DOI: https://doi.org/10.21203/rs.3.rs-1204698/v1

License: (c) (i) This work is licensed under a Creative Commons Attribution 4.0 International License. Read Full License 


\section{Abstract}

In view of proven antiplatelet activity of hydrazone group containing compounds, two series of hydrazone derivatives were synthesized by coupling appropriate aldehydes with phenyl hydrazine and Isonicotinic acid in the presence of distilled water and a catalytic amount of glacial acetic acid. All synthesized compounds were screened for their antiplatelet activity against induced aggregation by adenosine diphosphate (ADP) and arachidonic acid (AA). The results indicate that compounds in arylhydrazone group had shown satisfactory activity. Among them, 1-(3-methoxybenzylidene)-2-phenylhydrazine (1c), 2methoxy-4-(2-phenylhydrazono) methyl phenol (1 g), and 2-((2-phenylhydrazono) methyl)-1H-pyrrole (1h) were found to be the most potent antiplatelet compounds with $\mathrm{IC}_{50}$ less than $39 \mu \mathrm{M}$. Furthermore, the cell toxicity assay, (MTT test) indicates their noncytotoxic in various cell lines. None of the synthesized Nisonicotinohydrazide derivatives in this study excreted sufficient antiplatelet activity.

\section{Introduction}

Cardiovascular disorders (CVDs) are considered as the major causes of death among non-communicable diseases worldwide ${ }^{[1]}$. In 2017, 17.8 million people lost their lives due to cardiac and cerebral vascular diseases [2], and the mortality rate is expected to rise to 25 million by $2030{ }^{[3]}$. It is generally proven that platelet plays a key role in vascular haemostasis and thrombosis. While platelet aggregation is a vital process in normal hemostasis and clot formation for impeding bleeding, dysregulated platelet aggregation results in pernicious CVDs like transient ischemic attack and myocardial infarction (MI) ${ }^{[4]}$.

Amongst three classes of antithrombotic drugs (anticoagulant, antiplatelet, and thrombolytic), antiplatelet agents (such as aspirin, clopidogrel, or ticlopidine) are generally regarded as the first-line therapy for prophylaxis and treatment ${ }^{[5]}$. The most common antiplatelet drugs are categorized mainly into three groups; irreversible cyclooxygenase inhibitors, Glycoprotein IIB/IIIA inhibitors, and Adenosine diphosphate (ADP) receptor inhibitors ${ }^{[6]}$. Aspirin and triflusal impede cyclooxygenase I (COX-1) activity in transforming arachidonic acid to thromboxane $A_{2}\left(T X A_{2}\right)$. Clopidogrel, cangrelor, and prasugrel attach to $\mathrm{P}_{2} \mathrm{Y}_{12}$ ADP receptor and subsequently hinder the aggregation. Finally, abciximab, tirofiban, and eptifibatide that are IIB/IIIA antagonists and affect GPall $\beta$ (the most abundant receptor on the platelet surface), leading to inhibition of platelet aggregation ${ }^{[6]}$.

Although, these drugs have a substantial effect on alleviating thromboembolic diseases, they are flawed with some limitations comprising their potency, safety, and patients' adherence to treatment. Some of these drawbacks include; adverse effect on the digestive system (e.g. aspirin, ticlopidine, and clopidogrel) $[7,8]$, reduction in the number of platelet and white cells (e.g. aspirin) ${ }^{[9]}$, bleeding (e.g. tirofiban, clopidogrel, and aspirin) ${ }^{[10]}$, and variable responses (e.g. aspirin and clopidogrel) ${ }^{[11-14]}$.

Therefore, developing a new antiplatelet agent with little side effects and higher efficacy is an ongoing endeavor of researchers ${ }^{[10]}$. Compounds with hydrazone backbone have been reported to demonstrate 
diverse biological properties including anticonvulsant, antidepressant, antimalarial, antimicrobial, analgesic, and anti-inflammatory ${ }^{[15]}$. Interestingly, they can inhibit COX-1, which indicates that hydrazone derivatives are promising scaffold for designing an antiplatelet agent ${ }^{[16]}$.

The antiplatelet aggregation activity of heterocyclic acylhydrazone ${ }^{[17]}$ and $10-\mathrm{H}$-phenothiazine-1acylhydrazone ${ }^{[18]}$ have been reported. 2-hydrazinyl-1,3,4-thiadiazole derivatives were also reported as platelet aggregation inhibitor and some of them demonstrated $\mathrm{IC}_{50}$ values comparable to indomethacin when $A A$ used as platelet aggregation inducer ${ }^{[19]}$. We have reported the satisfactory antiplatelet activity of phenylhydrazones of indole-3-carbaldehyde (Figure 1, I) against platelet aggregation induced by AA [20]. Considering this background, in this study two groups of hydrazones were designed and synthesized according to the general procedure of Schiff bases synthesis ${ }^{[21,22]}$. A series of phenylhydrazones (1a-1j) was designed based on phenylhydrazone of indole-3-carbaldehyde (Figure 1, I). In order to further study of antiplatelet structure-activity relationship of hydrazones, indole ring replaced by heterocyclic aromatic rings and substituted benzene rings. The compounds $2 \mathrm{a}-2 \mathrm{l}$ were also designed by molecular simplification of N'-benzylidene-carbohydrazide-1H-pyrazolo[3,4-b]pyridines (Figure 1, II) which was designed and reported as antiplatelet agents by Lourençe et al. ${ }^{[23]}$. Finally, the antiplatelet activities of synthesized compounds against ADP and AA-induced aggregation were evaluated and effective compounds were assessed for their cell toxicity.

\section{Experimental Section Chemistry}

Chemical were purchased from Merck Chemical Company. Melting points $\left({ }^{\circ} \mathrm{C}\right)$ were determined by the Electrothermal IA9300 apparatus. (Cole-Parmer, Staffordshire, UK). Infrared spectra (IR) were recorded on a Perkin-Elmer 843 spectrometer employing potassium bromide $(\mathrm{KBr})$ as the diluent (PerkinElmer, UK). Proton nuclear magnetic resonance ( $\left.{ }^{1} \mathrm{H}-\mathrm{NMR}\right)$ spectra were acquired using the Bruker AVance series; AV300, AV-500, and AV-600 MHz Spectrometer and benchtop NMR spectrometer, the NMReady-60 NMRPro spectrometer. Dimethylsulfoxide (DMSO- $\mathrm{d}_{6}$ ) solution and tetramethylsaline were utilized as an internal standard. Coupling constant values $(J)$ are given in Hertz. Peak positions are expressed in parts per million (ס). Splitting patterns are as follows: $s$, singlet; $d$, doublet; $t$, triplet; $q$, quadruplet; $d d$, double doublet; m, multiplet. Mass spectra were obtained by Agilent 6410 Triple Quad LC MASS equipped with Electrospray lonization (ESI) and Agilent 7000 Triple Quad GC MASS provided Electronic Ionization (EI). (Santa Clara, United States) The development of all reactions was monitored by thin-layer chromatography (TLC) performed on aluminium sheets coated with silica and visualized by UV irradiation.

Synthesis of arylhydrazones derivitives $1 \mathrm{a}-1 \mathrm{j}$ 
Phenyl hydrazine $(0.005$ mole, $0.54 \mathrm{~g})$ was added to a stirring solution of corresponding aldehyde (0.005 mole) in distilled water $(10 \mathrm{~mL})$ and in the presence of $2 \mathrm{~mL}$ glacial acetic acid (as the catalyst). The mixture stirred at room temperature (RT). Once the solid was formed, completion of the reaction was confirmed by carrying out the TLC sample. Next, the precipitate was filtered off, washed with cold water, dried under vacuum, and recrystallized with appropriate solvents.

Compound $1 \mathbf{j}$ was synthesized in a different proportion of 0.005 moles of aldehyde (Glyoxal) and 0.01 mole of phenylhydrazine under the same condition described above.

N-(4-((2-phenylhydrazono)methyl)phenyl)acetamide (1a ) Yield: 70\%; m.p. 200; Recrystallization solvent: ethanol: water (1:1); ${ }^{1} \mathrm{HNMR}(600 \mathrm{MHz}$, [D6] DMSO): $\delta=10.194(\mathrm{~s}, 1 \mathrm{H} ; \mathrm{N}-\mathrm{NH}), 10.001(\mathrm{~s}, 1 \mathrm{H} ; \mathrm{NH}-$ CO), 7.787 (s, $1 \mathrm{H} ; \mathrm{C}=\mathrm{N}$ ), $7.59\left(\mathrm{~d},{ }^{3} \mathrm{~J}_{\mathrm{H}, \mathrm{H}}=8.4 \mathrm{~Hz}, 2 \mathrm{H}\right.$; Ph2-H3, Ph2-H5), 7.548(d, ${ }^{3} \mathrm{~J}_{\mathrm{H}, \mathrm{H}}=8.4 \mathrm{~Hz}, 2 \mathrm{H} ; \mathrm{Ph} 2-\mathrm{H} 2$, $\mathrm{Ph} 2-\mathrm{H} 6), 7.188\left(\mathrm{t},{ }^{3} J_{\mathrm{H}, \mathrm{H}}=7.2-7.8 \mathrm{~Hz}, 2 \mathrm{H}\right.$; Ph1-H3, Ph1-H5), 7.03( d, ${ }^{3} J_{\mathrm{H}, \mathrm{H}}=7.8 \mathrm{~Hz}, 2 \mathrm{H}$; Ph1-H2, Ph1-H6), 6.71 ( $\left.\mathrm{t},{ }^{3} \mathrm{~J}_{\mathrm{H}, \mathrm{H}}=7.2,1 \mathrm{H} ; \mathrm{Ph} 1-\mathrm{H} 4\right), 2.037 \mathrm{ppm}\left(\mathrm{s}, 3 \mathrm{H} ; \mathrm{CH}_{3}\right) ; \mathrm{IR}(\mathrm{KBr}): \tilde{v}=3265(\mathrm{~s})(\mathrm{NH}), 3183(\mathrm{~m})(\mathrm{CH}$, aromatic), $3098(\mathrm{~m})$ (CH, aliphatic), 1657 (vs) cm ${ }^{-1}$ (C=N); GC-MS (El) m/z (\%): 253 (100) [M+H] $]^{+}$; elemental analysis calcd (\%) for $\mathrm{C}_{15} \mathrm{H}_{15} \mathrm{~N}_{3} \mathrm{O}$ : C 71.13, $\mathrm{H}$ 5.97, N 16.59; found: C 71.21, H 5.96, N 16.53.

1-(3-nitrobenzylidene)-2-phenylhydrazine (1b ) Yield: 40\%; m.p. 122-125 ${ }^{\circ} \mathrm{C}$; Recrystallization solvent: ethanol: water (1:1); ${ }^{1} \mathrm{HNMR}(300 \mathrm{MHz},[\mathrm{D} 6] \mathrm{DMSO}): \delta=10.64(\mathrm{~s}, 1 \mathrm{H} ; \mathrm{NH}), 8.39(\mathrm{~s}, 1 \mathrm{H} ; \mathrm{Ph} 2-\mathrm{H} 2), 8.061(\mathrm{~m}$, $2 \mathrm{H} ; \mathrm{Ph} 2-\mathrm{H} 4, \mathrm{Ph} 2-\mathrm{H} 6), 7.92(\mathrm{~s}, 1 \mathrm{H} ; \mathrm{CH}=\mathrm{N}), 7.624\left(\mathrm{t},{ }^{3} \mathrm{~J}_{\mathrm{H}, \mathrm{H}}=8.1 \mathrm{~Hz}, 1 \mathrm{H} ; \mathrm{Ph} 2-\mathrm{H} 5\right), 7.216\left(\mathrm{t},{ }^{3} J_{\mathrm{H}, \mathrm{H}}=7.5 \mathrm{~Hz}, 2 \mathrm{H}\right.$; $\mathrm{Ph} 1-\mathrm{H} 3, \mathrm{Ph} 1-\mathrm{H} 5), 7.082\left(\mathrm{~d},{ }^{3} \mathrm{~J}_{\mathrm{H}, \mathrm{H}}=7.8 \mathrm{~Hz}, 2 \mathrm{H} ; \mathrm{Ph} 1-\mathrm{H} 2, \mathrm{Ph} 1-\mathrm{H} 6\right), 6.763 \mathrm{ppm}\left(\mathrm{t},{ }^{3} \mathrm{~J}_{\mathrm{H}, \mathrm{H}}=7.2 \mathrm{~Hz}, 1 \mathrm{H} ; \mathrm{Ph} 1-\mathrm{H} 4\right) ; \mathrm{IR}$ $(\mathrm{KBr}): \tilde{v}=3319(\mathrm{~s})(\mathrm{NH}), 1528(\mathrm{vs})(\mathrm{C}=\mathrm{N}), 1499$ and 1343(s) $\left(\mathrm{NO}_{2}\right), 692,739$ and $816(\mathrm{~m}) \mathrm{cm}^{-1}$ (metha substituted phenyl); GC-MS (EI) m/z (\%): $241(100)[M+H]^{+}$; elemental analysis calcd (\%) for $\mathrm{C}_{13} \mathrm{H}_{11} \mathrm{~N}_{3} \mathrm{O}_{2}$ : C 64.72, H 4.60, N 17.42; found: C 64.75, H 4.59, N 17.36.

1-(3-methoxybenzylidene)-2-phenylhydrazine (1c ) Yield: 50\%; m.p. $51-53^{\circ} \mathrm{C}$; Recrystallization solvent: ethanol: water (1:1); ${ }^{1} \mathrm{HNMR}(300 \mathrm{MHz}$, [D6] DMSO): $\delta=10.32(\mathrm{~s}, 1 \mathrm{H} ; \mathrm{NH}), 7.82(\mathrm{~s}, 1 \mathrm{H} ; \mathrm{CH}=\mathrm{N}), 7.27(\mathrm{~m}, 3 \mathrm{H}$; $\mathrm{Ph} 2-\mathrm{H} 2, \mathrm{Ph} 2-\mathrm{H} 5, \mathrm{Ph} 2-\mathrm{H} 6), 7.18-7.2(\mathrm{t}, 2 \mathrm{H} ; \mathrm{Ph} 1-\mathrm{H} 3, \mathrm{Ph} 1-\mathrm{H} 5), 7.06\left(\mathrm{~d},{ }^{3} \mathrm{~J}_{\mathrm{H}, \mathrm{H}}=7.5 \mathrm{~Hz}, 2 \mathrm{H}\right.$; Ph1-H2, Ph1-H6), $6.85\left(\mathrm{~d}, 1 \mathrm{H} ;{ }^{3} J_{\mathrm{H}, \mathrm{H}}=7.9 \mathrm{~Hz}, \mathrm{Ph} 2-\mathrm{H} 4\right), 6.738\left(\mathrm{t},{ }^{3} \mathrm{~J}_{\mathrm{H}, \mathrm{H}}=7.2 \mathrm{~Hz}, 1 \mathrm{H} ; \mathrm{Ph} 1-\mathrm{H} 4\right), 3.81 \mathrm{ppm}(\mathrm{s}, 3 \mathrm{H} ; \mathrm{OCH} 3) ; \mathrm{IR}(\mathrm{KBr})$ : $\tilde{v}=3310(\mathrm{~m})(\mathrm{NH}), 1588(\mathrm{vs})(\mathrm{C}=\mathrm{N}), 1263$ and $1033(\mathrm{~s})\left(\mathrm{Ar}-\mathrm{O}-\mathrm{CH}_{3}\right), 697,760$ and $792 \mathrm{~cm}^{-1}$ (m) (metha substituted phenyl); GC-MS (EI) m/z (\%): $226(100)[\mathrm{M}+\mathrm{H}]^{+}$; elemental analysis calcd (\%) for $\mathrm{C}_{14} \mathrm{H}_{14} \mathrm{~N}_{2} \mathrm{O}$ : C 74.31, H 6.24, N 12.38; found: C 74.3, H 6.23, N 12.4.

1-phenyl-2-(3, 4, 5-trimethoxybenzylidene)hydrazine (1d ) Yield: 30\%; m.p. 130-133 ${ }^{\circ} \mathrm{C}$; Recrystallization solvent: ethanol: water (1:1); ${ }^{1} \mathrm{HNMR}(300 \mathrm{MHz},[\mathrm{D} 6] \mathrm{DMSO}): \delta=10.27(\mathrm{~s}, 1 \mathrm{H} ; \mathrm{NH}), 7.77(\mathrm{~s}, 1 \mathrm{H} ; \mathrm{CH}=\mathrm{N})$, 7.18-7.23(t, ${ }^{3} \mathrm{~J}_{\mathrm{H}, \mathrm{H}}=7.5-7.8 \mathrm{~Hz}, 2 \mathrm{H}$; Ph1-H3, Ph1-H5), 7.06(d, $\left.{ }^{3} \mathrm{~J}_{\mathrm{H}, \mathrm{H}}=7.8 \mathrm{~Hz}, 2 \mathrm{H} ; \mathrm{Ph} 1-\mathrm{H} 2, \mathrm{Ph} 1-\mathrm{H} 6\right), 6.94(\mathrm{~s}, 2 \mathrm{H}$; $\mathrm{Ph} 2-\mathrm{H} 2, \mathrm{Ph} 2-\mathrm{H} 6), 6.728\left(\mathrm{t},{ }^{3} J_{\mathrm{H}, \mathrm{H}}=7.2 \mathrm{~Hz}, 1 \mathrm{H}\right.$; Ph1-H4), 3.82(s, 6H; OCH3, Meta, ortho), $3.67 \mathrm{ppm}(\mathrm{s}, 3 \mathrm{H}$; OCH3 para); IR (KBr): $\tilde{v}=3339(\mathrm{~m})(\mathrm{NH}), 3300(\mathrm{w})(\mathrm{CH}$, aromatic), $2975(\mathrm{w})(\mathrm{CH}$, aliphatic), 1632 (vs) 
$(\mathrm{C}=\mathrm{N}), 1249$ and $1024 \mathrm{~cm}^{-1}$ (s) $\left(\mathrm{Ar}-\mathrm{O}-\mathrm{CH}_{3}\right)$; GC-MS (EI) m/z (\%): 286.1 (100) [M+H] ; elemental analysis calcd (\%) for $\mathrm{C}_{16} \mathrm{H}_{18} \mathrm{~N}_{2} \mathrm{O}_{3}$ : C 67.12, $\mathrm{H} 6.34, \mathrm{~N}$ 9.78; found: C 67.2, H 6.33, N 9.77.

1-(2-chlorobenzylidene)-2-phenylhydrazine (1e ) Yield: $80 \%$; m.p. $81-85^{\circ} \mathrm{C}$; Recrystallization solvent: ethanol: water (1:1); ${ }^{1} \mathrm{HNMR}(300 \mathrm{MHz}$, [D6] DMSO): $\delta=10.67(\mathrm{~s}, 1 \mathrm{H} ; \mathrm{NH}), 8.19(\mathrm{~s}, 1 \mathrm{H} ; \mathrm{CH}=\mathrm{N}), 7.99-8.02(\mathrm{~d}$, $\left.{ }^{3} J_{\mathrm{H}, \mathrm{H}}=7.8 \mathrm{~Hz}, 1 \mathrm{H} ; \mathrm{Ph} 2-\mathrm{H} 3\right), 7.43\left(\mathrm{~d},{ }^{3} \mathrm{~J}_{\mathrm{H}, \mathrm{H}}=7.65 \mathrm{~Hz}, 1 \mathrm{H} ; \mathrm{Ph} 2-\mathrm{H} 6\right), 7.23\left(\mathrm{t},{ }^{3} \mathrm{~J}_{\mathrm{H}, \mathrm{H}}=7.5 \mathrm{~Hz}, 2 \mathrm{H} ; \mathrm{Ph} 1-\mathrm{H} 3, \mathrm{Ph} 1-\right.$ $\mathrm{H} 5), 7.33(\mathrm{~m}, 2 \mathrm{H} ; \mathrm{Ph} 2-\mathrm{H} 4, \mathrm{Ph} 2-\mathrm{H} 5), 7.08\left(\mathrm{~d},{ }^{3} J_{\mathrm{H}, \mathrm{H}}=7.5 \mathrm{~Hz}, 2 \mathrm{H} ; \mathrm{Ph} 1-\mathrm{H} 2, \mathrm{Ph}-\mathrm{H} 6\right), 6.77 \mathrm{ppm}\left(\mathrm{t},{ }^{3} J_{\mathrm{H}, \mathrm{H}}=7.2 \mathrm{~Hz}\right.$, 1H; Ph1-H4); IR (KBr): v =3116 (w) (NH) 1607 (s) (C=N), 765 (s) cm $\mathrm{cm}^{-1}$ (C-Cl); MS (ESI) m/z (\%): 231 (100) $[\mathrm{M}+\mathrm{H}]^{+}$; elemental analysis calcd (\%) for $\mathrm{C}_{13} \mathrm{H}_{11} \mathrm{ClN}_{2}$ : C 67.68, $\mathrm{H} 4.81, \mathrm{~N} 12.14$; found: $\mathrm{C} 67.67, \mathrm{H} 4.81, \mathrm{~N}$ 12.10 .

4-((2-phenylhydrazono)methyl)benzoic acid (1f ) Yield: 23\%; m.p. 221-225 ${ }^{\circ} \mathrm{C}$; Recrystallization solvent: ethanol: water (1:1); ${ }^{1} \mathrm{HNMR}(300 \mathrm{MHz}$, [D6] DMSO): $\delta=12.86(\mathrm{~s}, 1 \mathrm{H} ; \mathrm{COOH}), 10.58(\mathrm{~s}, 1 \mathrm{H} ; \mathrm{NH}), 7.91$ (d, $\left.{ }^{3} J_{\mathrm{H}, \mathrm{H}}=8.4 \mathrm{~Hz}, 2 \mathrm{H} ; \mathrm{Ph} 2-\mathrm{H} 3, \mathrm{Ph} 2-\mathrm{H} 5\right), 7.886(\mathrm{~s}, 1 \mathrm{H} ; \mathrm{CH}=\mathrm{N}), 7.73\left(\mathrm{~d},{ }^{3} \mathrm{~J}_{\mathrm{H}, \mathrm{H}}=8.4 \mathrm{~Hz}, 2 \mathrm{H} ; \mathrm{Ph} 2-\mathrm{H} 2, \mathrm{Ph} 2-\mathrm{H} 6\right), 7.23(\mathrm{t}$, $\left.{ }^{3} J_{\mathrm{H}, \mathrm{H}}=7.8 \mathrm{~Hz}, 2 \mathrm{H} ; \mathrm{Ph} 1-\mathrm{H} 3, \mathrm{Ph} 1-\mathrm{H} 5\right), 7.09\left(\mathrm{~d},{ }^{3} \mathrm{~J}_{\mathrm{H}, \mathrm{H}}=7.8 \mathrm{~Hz}, 2 \mathrm{H} ; \mathrm{Ph} 1-\mathrm{H} 2, \mathrm{Ph} 1-\mathrm{H} 6\right), 6.77 \mathrm{ppm}\left(\mathrm{t},{ }^{3} \mathrm{~J}_{\mathrm{H}, \mathrm{H}}=7.2 \mathrm{~Hz}\right.$, 1H; Ph1-H4); IR (KBr): v =3323 (br) (OH and NH), $3073(\mathrm{w})(\mathrm{CH}$, aromatic), $1712(\mathrm{~m})(\mathrm{C}=0), 1680(\mathrm{~s})(\mathrm{C}=\mathrm{N})$ 767 and 699 (s) $\mathrm{cm}^{-1}$ (mono substituted phenyl); MS (ESI) m/z (\%): 241 (100) [M+H] ${ }^{+}$; elemental analysis calcd (\%) for $\mathrm{C}_{14} \mathrm{H}_{12} \mathrm{~N}_{2} \mathrm{O}_{2}$ : C 69.99, H 5.03, N 11.66; found: C 70.07, H 5.04, N 11.62.

2-methoxy-4-(2-phenylhydrazono)methyl phenol (1g ) Yield: $42 \%$; m.p. $65-66^{\circ} \mathrm{C}\left({ }^{(i t}{ }^{[26]}\right.$ m.p. $\left.64-65^{\circ} \mathrm{C}\right)$; Recrystallization solvent: methanol: water (3:2); ${ }^{1} \mathrm{HNMR}(100 \mathrm{MHz},[\mathrm{D} 6] \mathrm{CDCl} 3)$ : $\delta=7.5-7.75(\mathrm{~s}, 1 \mathrm{H}$; $\mathrm{CH}=\mathrm{N})$, 6.75-7.40 (m, 10H; $\mathrm{Ar} \mathrm{H}, \mathrm{CH}=\mathrm{N}$ and $\mathrm{NH})$, 5.80-5.85 (s, 1H; OH), 3.85-4.00 (s, 3H; CH3); IR (KBr): $\tilde{v}=3532(\mathrm{br})(\mathrm{OH}), 3342(\mathrm{~m})(\mathrm{NH}), 3083(\mathrm{w})(\mathrm{CH}$, aromatic), $2969(\mathrm{w})(\mathrm{CH}$, aliphatic), $1611(\mathrm{vs})(\mathrm{C}=\mathrm{N})$, 1276 and $1046 \mathrm{~cm}^{-1}$ (s) $\left(\mathrm{Ar}-\mathrm{O}-\mathrm{CH}_{3}\right)$; MS (ESI) m/z (\%): 243 (100) [M+H] $]^{+}$; elemental analysis calcd (\%) for $\mathrm{C}_{14} \mathrm{H}_{14} \mathrm{~N}_{2} \mathrm{O}_{2}$ : C 69.41, H 5.82, N 11.56; found: C 96.41, H 5.81, N 11.6.

2-((2-phenylhydrazono)methyl)-1H-pyrrole (1h ) Yield: 70\%; m.p. 137-140 ${ }^{\circ} \mathrm{C}$; Recrystallization solvent: ethanol; ${ }^{1} \mathrm{HNMR}(600 \mathrm{MHz}$, [D6] DMSO): $\delta=11.09$ (s, $1 \mathrm{H} ; \mathrm{NH}$ pyrrole), $7.71(\mathrm{~s}, 1 \mathrm{H} ; \mathrm{CH}=\mathrm{N}), 9.87(\mathrm{~s}, 1 \mathrm{H} ; \mathrm{NH})$, $7.157\left(\mathrm{t},{ }^{3} \mathrm{~J}_{\mathrm{H}, \mathrm{H}}=7.8 \mathrm{~Hz}, 2 \mathrm{H} ; \mathrm{Ph} 1-\mathrm{H} 3, \mathrm{Ph} 1-\mathrm{H} 5\right), 7.027$ (d, 2H; $\left.{ }^{3} \mathrm{~J}_{\mathrm{H}, \mathrm{H}}=7.8 \mathrm{~Hz}, \mathrm{Ph} 1-\mathrm{H} 2, \mathrm{Ph} 1-\mathrm{H} 6\right), 6.799(\mathrm{~s}, 1 \mathrm{H} ; \mathrm{H} 3$ pyrrole), $6.66\left(\mathrm{t}, 1 \mathrm{H} ;{ }^{3} \mathrm{~J}_{\mathrm{H}, \mathrm{H}}=7.2 \mathrm{~Hz}, \mathrm{Ph} 1-\mathrm{H} 4\right), 6.23(\mathrm{~s}, 1 \mathrm{H} ; \mathrm{H} 4$ pyrrole), $6.05 \mathrm{ppm}$ (d, $1 \mathrm{H} ; \mathrm{H} 5$ pyrrole); IR (KBr): $\tilde{v}=3432(\mathrm{~m})(\mathrm{NH}), 3327(\mathrm{w})\left(\mathrm{CH}\right.$, aromatic), $1600(\mathrm{vs})(\mathrm{C}=\mathrm{N}) 749$ and $695(\mathrm{~s}) \mathrm{cm}^{-1}$ (mono substituted phenyl); MS (ESI) m/z (\%): 185 (100) $[\mathrm{M}+\mathrm{H}]^{+}$; elemental analysis calcd (\%) for $\mathrm{C}_{11} \mathrm{H}_{11} \mathrm{~N}_{3}$ : C 71.33, H 5.99, N 22.69; found: C 71.29, H 5.99, N 22.72.

3-((2-phenylhydrazono) methyl)-1H-indole (1i ) Yield: 70\%; m.p. 200-204 ${ }^{\circ} \mathrm{C}$; Recrystallization solvent: methanol: water (3:2); ${ }^{1} \mathrm{HNMR}(300 \mathrm{MHz}$, [D6] DMSO): $\delta=11.29$ (s, $1 \mathrm{H} ; \mathrm{NH}$ indole), 9.79(s,1H; NH), 8.244(d, $1 \mathrm{H} ; \mathrm{J}=6, \mathrm{H} 5$ indole), 8.10(s, $1 \mathrm{H}, \mathrm{C}=\mathrm{N}), 7.61\left(\mathrm{~d}, 1 \mathrm{H} ; \mathrm{H} 6\right.$ indole), $7.41\left(\mathrm{~d},{ }^{3} \mathrm{~J}_{\mathrm{H}, \mathrm{H}}=6.15 \mathrm{~Hz}, 1 \mathrm{H} ; \mathrm{H} 4\right.$ indole), 7.17(m, 4H; Ph1-H3, Ph1-H5,H3 indole, H9 indole), 7.03(d, J=7.8 Hz, 2H, Ph1-H2, Ph1-H6), 6.67 $\operatorname{ppm}\left(\mathrm{t},{ }^{3} \mathrm{~J}_{\mathrm{H}, \mathrm{H}}=7.2 \mathrm{~Hz}, 1 \mathrm{H} ; \mathrm{Ph} 1-\mathrm{H} 4\right) ; \mathrm{IR}(\mathrm{KBr}): \tilde{v}=3456(\mathrm{~s})(\mathrm{NH}), 3312(\mathrm{~m})(\mathrm{CH}$, aromatic), 1610 (vs) (C=N), 
$1490(\mathrm{~m})\left(\mathrm{NH}\right.$, indole ring), 782 (vs) $\mathrm{cm}^{-1}$ (CH out of plane bending, indole ring); GC-MS (EI) m/z (\%): 236 (100) $[\mathrm{M}+\mathrm{H}]^{+}$; elemental analysis calcd (\%) for $\mathrm{C}_{15} \mathrm{H}_{13} \mathrm{~N}_{3}$ : C 76.57, H 5.57, N 17.86; found: C 76.58, H 5.58, N 17.84.

1,2-bis(2-phenylhydrazono)ethane (1j ) Yield: $13 \%$; m.p. 179-179.9 $9^{\circ} \mathrm{C}$; Recrystallization solvent: ethanol; ${ }^{1} \mathrm{HNMR}\left(600 \mathrm{MHz}\right.$, [D6] DMSO): $\delta=10.35(\mathrm{~s}, 1 \mathrm{H} ; \mathrm{NH}), 7.63(\mathrm{~s}, 1 \mathrm{H} ; \mathrm{CH}=\mathrm{N}), 7.19\left(\mathrm{t},{ }^{3} \mathrm{~J}_{\mathrm{H}, \mathrm{H}}=7.2-7.8 \mathrm{~Hz}, 2 \mathrm{H}\right.$; Ph1$\mathrm{H} 3$, Ph1-H5), 6.97(d, $\left.{ }^{3} J_{\mathrm{H}, \mathrm{H}}=7.8 \mathrm{~Hz}, 2 \mathrm{H} ; \mathrm{Ph} 1-\mathrm{H} 2, \mathrm{Ph} 1-\mathrm{H} 6\right), 6.73 \mathrm{ppm}\left(\mathrm{t},{ }^{3} \mathrm{~J}_{\mathrm{H}, \mathrm{H}}=7.2 \mathrm{~Hz}, 1 \mathrm{H} ; \mathrm{Ph} 1-\mathrm{H} 4\right) ; \mathrm{IR}(\mathrm{KBr})$ : $\tilde{v}=3319(\mathrm{~m})(\mathrm{NH}), 3043(\mathrm{w})\left(\mathrm{CH}\right.$, aromatic), $1604(\mathrm{vs})(\mathrm{C}=\mathrm{N}) 767$ and $698(\mathrm{~s}) \mathrm{cm}^{-1}$ (mono substituted phenyl); GC-MS (EI) m/z (\%): $\mathrm{m} / \mathrm{z}=238(60)[\mathrm{M}+\mathrm{H}]^{+}$; elemental analysis calcd (\%) for $\mathrm{C}_{14} \mathrm{H}_{14} \mathrm{~N}_{4}: \mathrm{C} 70.57$, H 5.92, N 23.51; found: C 70.55, H 5.92, N 23.53.

\section{Synthesis of N-isonicotinohydrazide derivatives 2a-2l}

Isonicotinic acid hydrazide ( 0.005 mole, $0.68 \mathrm{~g})$ was added slowly to a stirring solution of appropriate aldehyde $(0.005$ mole) in distilled water $(10 \mathrm{~mL})$ and in presence of $2 \mathrm{~mL}$ glacial acetic acid (as catalyst). The mixture stirred at room temperature (RT). Once the solid was formed, completion of the reaction was confirmed by carrying out the TLC sample. Next, the precipitate was filtered off, washed with cold water, dried under vacuum, and recrystallized with appropriate solvents.

$\mathrm{N}$-(4-((2-isonicotinoylhydrazono)methyl)phenyl)acetamide (2a ) Yield: $91 \%$; mp $288-292^{\circ} \mathrm{C}$;

Recrystallization solvent: ethanol; ${ }^{1} \mathrm{HNMR}(60 \mathrm{MHz}$, [D6] DMSO): $\delta=12.0(\mathrm{~s}, 1 \mathrm{H} ; \mathrm{N}-\mathrm{NH}), 10.2(\mathrm{~s}, 1 \mathrm{H} ; \mathrm{NH}$ amide), 7.5-8.5 (m, 9H; phenyl and pyridine $\mathrm{H}$, and $\mathrm{CH}=\mathrm{N}), 2 \mathrm{ppm}(\mathrm{s}, 3 \mathrm{H} ; \mathrm{CH} 3) ; \mathrm{IR}(\mathrm{KBr}): \tilde{v}=3303(\mathrm{~s})(\mathrm{NH}$, amide), 3035 (m) (CH, aromatic), 2926 (w) (CH, aliphatic), 1659 (vs) (C=O amide), 1582 (vs) (C=N), 843 (s) $\mathrm{cm}^{-1}$ (CH out of plane bending, 4-substituted pyridine); MS (ESI) m/z (\%): 283 (100) [M+H] ; elemental analysis calcd (\%) for $\mathrm{C}_{15} \mathrm{H}_{14} \mathrm{~N}_{4} \mathrm{O}_{2}$ : C 63.82, H 5.00, N 19.85; found: C 63.81, H 4.99, N 19.84.

$N^{\prime}$-(3-nitrobenzylidene)isonicotinohydrazide (2b ) Yield: 70\%; m.p. $227-230^{\circ} \mathrm{C}$; Recrystallization solvent: ethanol; ${ }^{1} \mathrm{HNMR}(500 \mathrm{MHz},[\mathrm{D} 6] \mathrm{DMSO}): \delta=12.32(\mathrm{~s}, 1 \mathrm{H} ; \mathrm{NH}), 8.79-8.80\left(\mathrm{~d},{ }^{3} \mathrm{~J}_{\mathrm{H}, \mathrm{H}}=4.5 \mathrm{~Hz}, 2 \mathrm{H}\right.$; pyridine-H3,$\mathrm{H} 5), 8.573(\mathrm{~s}, 2 \mathrm{H} ; \mathrm{CH}=\mathrm{N}, \mathrm{Ph}-\mathrm{H} 2), 8.27-8.29\left(\mathrm{~d},{ }^{3} \mathrm{~J}_{\mathrm{H}, \mathrm{H}}=8 \mathrm{~Hz}, 1 \mathrm{H} ; \mathrm{Ph}-\mathrm{H} 4\right), 8.17-8.19\left(\mathrm{~d},{ }^{3} J_{\mathrm{H}, \mathrm{H}}=8 \mathrm{~Hz}, 1 \mathrm{H} ; \mathrm{Ph}-\mathrm{H} 6\right)$, 7.82-7.83(d, ${ }^{3} J_{\mathrm{H}, \mathrm{H}}=4.5 \mathrm{~Hz}, 2 \mathrm{H}$; pyridine-H2,-H6), 7.75-7.81 ppm (t, $\left.{ }^{3} \mathrm{~J}_{\mathrm{H}, \mathrm{H}}=8 \mathrm{~Hz}, 1 \mathrm{H} ; \mathrm{Ph}-\mathrm{H} 5\right) ; \mathrm{IR}(\mathrm{KBr})$ : $\tilde{v}=3423(\mathrm{~s})(\mathrm{NH}$, amide), $3208(\mathrm{~m})(\mathrm{CH}$, aromatic), $3034(\mathrm{~m})(\mathrm{CH}$, aliphatic), 1663 (vs) (C=0 amide), 1525 (vs) (C=N), 1500 and $1350(\mathrm{~m})\left(\mathrm{NO}_{2}\right) 850(\mathrm{w}) \mathrm{cm}^{-1}$ (CH out of plane bending, 4-substituted pyridine); MS (ESI) m/z (\%): 271 (100) [M+H] ; Anal.Calcd for $\mathrm{C}_{13} \mathrm{H}_{10} \mathrm{~N}_{4} \mathrm{O}_{3}$ : C 57.78, H 3.73, N 20.73; found: C 57.79, H $3.72, \mathrm{~N} 20.74$.

$N^{\prime}-\left(3-\right.$-methoxybenzylidene)isonicotinohydrazide (2c ) Yield: 32\%; m.p. 218-222 ${ }^{\circ} \mathrm{C}$; Recrystallization solvent: ethanol; ${ }^{1} \mathrm{HNMR}(60 \mathrm{MHz}$, [D6] DMSO): $\delta=12.2(\mathrm{~s}, 1 \mathrm{H} ; \mathrm{N}-\mathrm{NH}), 7.5-8.5(\mathrm{~m}, 9 \mathrm{H}$; phenyl and pyridine $\mathrm{H}, \mathrm{CH}=\mathrm{N}), 3 \mathrm{ppm}\left(\mathrm{s}, 3 \mathrm{H} ; \mathrm{OCH}_{3}\right) ; \mathrm{IR}(\mathrm{KBr}): \tilde{v^{\sim}=3549}$ (s) ( $\mathrm{NH}$, amide), $3182(\mathrm{w})(\mathrm{CH}$, aromatic), 3058 (w) (CH, aliphatic), 1659 (vs) (C=O, amide), 1588 (s) (C=N), 1290 and 1053 (s) $\left(A r-0-C_{3}\right), 859 \mathrm{~cm}^{-1}(\mathrm{CH}$ 
out of plane bending, 4-substituted pyridine); MS (ESI) m/z (\%): 260 (100) [M+H] ${ }^{+}$; elemental analysis calcd (\%) for $\mathrm{C}_{14} \mathrm{H}_{13} \mathrm{~N}_{3} \mathrm{O}_{2}$ : C 65.87, H 5.13, N 16.46; found: C 65.88, H 5.12, N 16.47.

$N^{\prime}-\left(2-\right.$ nitrobenzylidene)isonicotinohydrazide (2d) Yield: 70\%; m.p. $225-229^{\circ} \mathrm{C}\left(\right.$ Lit. $^{[27]} \mathrm{m}$. p. $\left.226-228^{\circ} \mathrm{C}\right)$; Recrystallization solvent: ethanol; ${ }^{1} \mathrm{HNMR}(60 \mathrm{MHz}$, [D6] DMSO): $\delta=12.1(\mathrm{~s}, 1 \mathrm{H} ; \mathrm{N}-\mathrm{NH}), 7.5-8.5 \mathrm{ppm}(\mathrm{m}$, 9H; phenyl and pyridine $\mathrm{H}, \mathrm{CH}=\mathrm{N})$; IR (KBr): $v^{\sim}=3431(\mathrm{~s})(\mathrm{NH}$, amide), $3167(\mathrm{~m})(\mathrm{CH}$, aromatic), $2994(\mathrm{~m})$ (CH, aliphatic), 1655 (vs) (C=0, amide), 1570 (s) (C=N), 1513 and $1346(\mathrm{~s})\left(\mathrm{NO}_{2}\right), 844(\mathrm{w}) \mathrm{cm}^{-1}$ (CH out of plane bending, 4-substituted pyridine); MS (ESI) m/z (\%): 271 (100) [M+H] $]^{+}$; elemental analysis calcd (\%) for $\mathrm{C}_{13} \mathrm{H}_{10} \mathrm{~N}_{4} \mathrm{O}_{3}$ : C 57.78, H 3.73, N 20.73; found: C 57.79, H 3.72, N 20.8.

$N^{\prime}$-(4-hydroxy-3-methoxybenzylidene)isonicotinohydrazide (2e ) Yield: 30\%; m.p. 217-220 C;

Recrystallization solvent: 1-propanol; ${ }^{1} \mathrm{H}$ NMR $(300 \mathrm{MHz}$, [D6] DMSO): $\delta=11.86(\mathrm{~s}, 1 \mathrm{H}$; -NH-N=), 8.76 (d, ${ }^{3} J_{\mathrm{H}, \mathrm{H}}=4.3 \mathrm{~Hz}, 2 \mathrm{H}$; pyridine), $8.34(\mathrm{~s}, 1 \mathrm{H} ;-\mathrm{N}=\mathrm{C}-\mathrm{H}), 7.80$ (d, 2H; pyridine, J = $\left.3.9 \mathrm{~Hz}\right), 7.32(\mathrm{~s}, 1 \mathrm{H}$; benzylidene), $7.09\left(\mathrm{~d},{ }^{3} \mathrm{~J}_{\mathrm{H}, \mathrm{H}}=8.2 \mathrm{~Hz}, 2 \mathrm{H}\right.$; benzylidene), $5.08\left(\mathrm{~s}, 1 \mathrm{H} ; \mathrm{OH}, \mathrm{D}_{2} \mathrm{O}\right.$ exchangeable), $3.82 \mathrm{ppm}$ (s, $\left.3 \mathrm{H} ; \mathrm{CH}_{3}\right) i^{[28]} \mathrm{IR}(\mathrm{KBr}): \tilde{v}=3527(\mathrm{~m})(\mathrm{OH}), 3459(\mathrm{~m})$ (NH amide), $3075(\mathrm{~m})$ (CH aromatic), 1669 (s) (C=0, amide), 1593 (s) (C=N), 1285 (s) $\mathrm{cm}^{-1}$ (Ar-O-C); MS (ESI) m/z (\%): 272 (100) [M+H] ; elemental analysis calcd (\%) for $\mathrm{C}_{14} \mathrm{H}_{13} \mathrm{~N}_{3} \mathrm{O}_{3}$ : C 61.99, H 4.83, N 15.49; found: C 61.97, H 4.82, N 15.51.

N'-((1H-pyrrol-2-yl) methylene)isonicotinohydrazide (2f) Yield: $26 \%$; m.p. $232-233^{\circ} \mathrm{C}$ (Lit. ${ }^{[29]}$ m.p.. 230$\left.232^{\circ} \mathrm{C}\right)$; Recrystallization solvent: 1-propanol; ${ }^{1} \mathrm{HNMR}(60 \mathrm{MHz},[\mathrm{D} 6] \mathrm{DMSO}): \delta=12(\mathrm{~s}, 2 \mathrm{H} ; \mathrm{N}-\mathrm{NH}), 7.5-8.5$ ppm (m, 8H; pyrrole and pyridine $\mathrm{H}, \mathrm{CH}=\mathrm{N})$; IR $(\mathrm{KBr}): \tilde{v}=3401(\mathrm{~m})(\mathrm{NH}$, amide), $3211(\mathrm{~m})(\mathrm{CH}$, aromatic), $2973(\mathrm{~m})\left(\mathrm{CH}\right.$, aliphatic), 1660 (s) (C=O, amide), 1610 (s) (C=N), $845(\mathrm{w}) \mathrm{cm}^{-1}$ (CH out of plane bending, 4substituted pyridine); MS (ESI) m/z (\%): 215 (100) $[\mathrm{M}+\mathrm{H}]^{+}$; elemental analysis calcd (\%) for $\mathrm{C}_{11} \mathrm{H}_{10} \mathrm{~N}_{4} \mathrm{O}: \mathrm{C}$ 61.67, H 4.71, N 26.15; found: C 61.68, H 4.72, N 26.14.

$N^{\prime}$-((E)-3-phenylallylidene)isonicotinohydrazide (2g ) Yield: 40\%; m.p. 199-203 C; Recrystallization solvent: ethanol; ${ }^{1} \mathrm{HNMR}(60 \mathrm{MHz}$, [D6] DMSO): $\delta=12(\mathrm{~s}, 1 \mathrm{H} ; \mathrm{N}-\mathrm{NH}), 7-9 \mathrm{ppm}(\mathrm{m}, 12 \mathrm{H}$; phenyl and pyridine

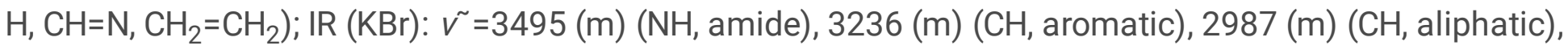
1672 (s) (C=0, amide), 1645 (s) (C=N), $843(\mathrm{w}) \mathrm{cm}^{-1}$ ( $\mathrm{CH}$ out of plane bending, 4-substituted pyridine); MS (ESI) $\mathrm{m} / \mathrm{z}$ (\%): $252(100)[\mathrm{M}+\mathrm{H}]^{+}$; elemental analysis calcd (\%) for $\mathrm{C}_{15} \mathrm{H}_{13} \mathrm{~N}_{3} \mathrm{O}: \mathrm{C}$ 71.70, H 5.21, N 16.72; found: C 71.7, H 5.21, N 16.74 .

$N^{\prime}$-(pyridin-3-y/methylene)isonicotinohydrazide (2h ) Yield: 34\%; m.p. 239-243ํㅜ ${ }^{\circ}$; Recrystallization solvent: 1-propanol; ${ }^{1} \mathrm{HNMR}$ (60 MHz, [D6] DMSO): $\delta=12.2$ (s, 1H; N-NH), 7.5 - 8.5 ppm (m. 9H; pyridine $\mathrm{H}, \mathrm{CH}=\mathrm{N})$; IR $(\mathrm{KBr}): \tilde{v}=3192(\mathrm{~m})(\mathrm{NH}$, amide), $3009(\mathrm{~m})(\mathrm{CH}$, aromatic), $2936(\mathrm{~m})(\mathrm{CH}$, aliphatic), $1678(\mathrm{vs})$ $\left(\mathrm{C}=0\right.$, amide), $1594(\mathrm{~m})(\mathrm{C}=\mathrm{N}), 849(\mathrm{~m})\left(\mathrm{CH}\right.$ out of plane bending, 4-substituted pyridine), $822(\mathrm{w}) \mathrm{cm}^{-1}$ (CH out of plane bending, 3-substituted pyridine); MS (ESI) m/z (\%): 227 (100) [M+H] ${ }^{+}$; elemental analysis calcd (\%) for $\mathrm{C}_{12} \mathrm{H}_{10} \mathrm{~N}_{4} \mathrm{O}$ : C 63.71, H 4.46, N 24.76; found: C 63.83, H 4.45, N 24.67. 
$N^{\prime}$-(pyridin-4-y/methylene)isonicotinohydrazide (2i ) Yield: 34\%; m.p. $231-235^{\circ} \mathrm{C}$; Recrystallization solvent: 1-propanol; ${ }^{1} \mathrm{HNMR}(60 \mathrm{MHz}$, [D6] DMSO): $\delta=12.2$ (s, 1H; N-NH), $7.5-8.5$ ppm (m, 9H; pyridine $\mathrm{H}, \mathrm{CH}=\mathrm{N})$; IR (KBr): $\tilde{v}=3480(\mathrm{~m})(\mathrm{NH}$, amide), $3196(\mathrm{~m})(\mathrm{CH}$, aromatic), $3017(\mathrm{~m})(\mathrm{CH}$, aliphatic), $1669(\mathrm{~s})(\mathrm{C}=0$, amide), 1574 (s) (C=N), $836(\mathrm{w}) \mathrm{cm}^{-1}$ (CH out of plane bending, 4-substituted pyridine); MS (ESI) m/z (\%): $227(100)[\mathrm{M}+\mathrm{H}]^{+}$; elemental analysis calcd (\%) for $\mathrm{C}_{12} \mathrm{H}_{10} \mathrm{~N}_{4} \mathrm{O}$ : C 63.71, H 4.46, N 24.76; found: $\mathrm{C} 63.72, \mathrm{H}$ 4.47, N 24.76.

$N^{\prime}$-(4-nitrobenzylidene)isonicotinohydrazide (2j ) Yield: 93\%; m.p. $195-200^{\circ} \mathrm{C}$; Recrystallization solvent: ethanol; ${ }^{1} \mathrm{HNMR}(60 \mathrm{MHz}$, [D6] DMSO): $\delta=12.2$ (s, 1H; N-NH), $7.5-8.5 \mathrm{ppm}(\mathrm{m}, 9 \mathrm{H}$; phenyl and pyridine $\mathrm{H}, \mathrm{CH}=\mathrm{N})$; IR (KBr): v =3196 (w) ( $\mathrm{NH}$, amide), $3000(\mathrm{w})(\mathrm{CH}$, aromatic), 2904 (w) (CH, aliphatic), 1684 (vs) (C=0, amide), $1570(\mathrm{~s})(\mathrm{C}=\mathrm{N}), 1514$ and $1342(\mathrm{vs})\left(\mathrm{NO}_{2}\right), 856(\mathrm{~m}) \mathrm{cm}^{-1}$ ( $\mathrm{CH}$ out of plane bending, 4substituted pyridine); MS (ESI) m/z (\%): 271 (100) $[\mathrm{M}+\mathrm{H}]^{+}$; elemental analysis calcd (\%) for $\mathrm{C}_{13} \mathrm{H}_{10} \mathrm{~N}_{4} \mathrm{O}_{3}$ : C 57.78, H 3.73, N 20.73; found: C 57.77, H 3.73, N 20.72.

$N^{\prime}$-benzylideneisonicotinohydrazide (2k ) Yield: $60 \%$; m.p. $198-200^{\circ} \mathrm{C}$; Recrystallization solvent: ethanol;

${ }^{1} \mathrm{H}-\mathrm{NMR}(60 \mathrm{MHz},[\mathrm{D} 6] \mathrm{DMSO}): \delta=12.06(\mathrm{~s}, 1 \mathrm{H} ; \mathrm{N}-\mathrm{H}), 8.80\left(\mathrm{~d},{ }^{3} \mathrm{~J}_{\mathrm{H}, \mathrm{H}}=6 \mathrm{~Hz}, 2 \mathrm{H}\right.$; aromatic H), $8.48(\mathrm{~s}, 1 \mathrm{H}$; azomethine), 7.84-7.74 (m, 4H; aromatic), 7.49-7.46 ppm (m, 3H; aromatic); ${ }^{[30]} \mathrm{IR}(\mathrm{KBr}): v^{\sim}=3408(\mathrm{~m})$ $(\mathrm{NH}$, amide), $3197(\mathrm{~m})(\mathrm{CH}$, aromatic), $3031(\mathrm{~m})(\mathrm{CH}$, aliphatic), $1668(\mathrm{~s})(\mathrm{C}=\mathrm{O}$, amide), $1575(\mathrm{~m})(\mathrm{C}=\mathrm{N})$, 847 (w) (CH out of plane bending, 4-substituted pyridine), 707 and $690(\mathrm{~m}) \mathrm{cm}^{-1}$ (mono substitution phenyl group); $\mathrm{MS}$ (ESI) m/z (\%): $226(100)[\mathrm{M}+\mathrm{H}]^{+}$; elemental analysis calcd (\%) for $\mathrm{C}_{13} \mathrm{H}_{11} \mathrm{~N}_{3} \mathrm{O}: \mathrm{C} 69.32$, H 4.92, N 18.66; found: C 69.33, H 4.91, N 18.65 .

$N^{\prime}-\left(\left[1,1^{\prime}-\right.\right.$ biphenyl]-4-ylmethylene)isonicotinohydrazide (2I ) Yield: $70 \%$; m.p. $218-221^{\circ} \mathrm{C} ;{ }^{1} \mathrm{HNMR}(60 \mathrm{MHz}$, [D6] DMSO): $\delta=12(\mathrm{~s}, 1 \mathrm{H} ; \mathrm{N}-\mathrm{NH}), 8.5(\mathrm{~m}, 3 \mathrm{H}$; pyridine-H3, $-\mathrm{H} 5, \mathrm{CH}=\mathrm{N}), 7.5 \mathrm{ppm}(\mathrm{m}, 11 \mathrm{H}$; pyridine-H2, $-\mathrm{H} 6$,

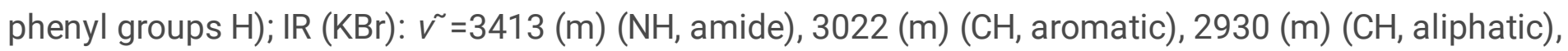
$1666(\mathrm{~s})\left(\mathrm{C}=0\right.$, amide), $1597(\mathrm{~m})(\mathrm{C}=\mathrm{N}), 839(\mathrm{w}) \mathrm{cm}^{-1}$ ( $\mathrm{CH}$ out of plane bending, 4-substituted pyridine); MS (ESI) m/z (\%): $302(100)[\mathrm{M}+\mathrm{H}]^{+}$; elemental analysis calcd (\%) for $\mathrm{C}_{19} \mathrm{H}_{15} \mathrm{~N}_{3} \mathrm{O}: \mathrm{C} 75.73, \mathrm{H} 5.02, \mathrm{~N}$ 13.94; found: C 75.74, H 5.03, N 13.92.

\section{In vitro study of anti-platelet aggregation activity}

The anti-platelet aggregation activity was determined in vitro using the human plasma. Blood was withdrawn from healthy volunteers (non-smoker) with no history of drug-consumption within fifteen days before the blood drawn. Platelet-rich plasma (PRP) was obtained by centrifugation of citrated whole blood (9:1) at $1000 \mathrm{rpm}$ for 8 minutes. The platelet-poor plasma (PPP) was prepared by centrifugation of the remained layer at $3000 \mathrm{rpm}$ for 15 minutes and was collected the upper layer (used as blank). In order to adjust the platelet, count to an appropriate amount, PPP was added to PRP and diluted to the number of $250000 \mathrm{plt} / \mu \mathrm{L}$. Test compounds dissolved in DMSO were added to PRP samples and incubated for 5 minutes at $37^{\circ} \mathrm{C}$. Following the addition of $\mathrm{ADP}(5 \mu \mathrm{L})$ or $\mathrm{AA}(18 \mu \mathrm{L})(1.25 \mathrm{mg} / \mathrm{mL})$, the variation and changes in aggregation pattern and platelet shape and aggregation were observed. 
DMSO was utilized as negative control while indomethacin or aspirin were the standard drugs. The formula stated below was used to calculate the platelet aggregation inhibition (\%):

Inhibition $(\%)=[1-(D / S)] * 100$

In the preceding formula $D$ is defined as the platelet aggregation in the presence of the test compound, while $S$ is the platelet aggregation in solvent ${ }^{[31,32]} . I_{50}$ was defined as the concentration that inhibits the platelet aggregation by $50 \%$ and all of the synthesized compounds were screened at the concentration of $1 \mathrm{mM}$.

\section{Cell viability test (MTT Test)}

To measure cell viability in the presence of the effective synthesized compounds, the MTT test was performed by methods previously described ${ }^{[33,34]}$. To prepare the MTT solution, first 3-(4,5Dimethylthiazol-2-yl)-2,5-diphenyltetrazolium bromidefor (MTT) was dissolved in Dulbecco Phosphate Buffer Saline (DPBS) with pH equals to 7.4 then the solution was filtered off $(0.2 \mu \mathrm{M}$ filter size).

Subsequently, the cells and selected derivative were placed in 96-well plates and incubated. Afterward, 10 $\mu \mathrm{L}$ of MTT solution was added to each cell and finally incubated for 1 to 4 hours at $37^{\circ} \mathrm{C}$. To dissolve formazan crystals $100 \mu \mathrm{L}$ of solubilization solution (Dimethylformamide $40 \% \mathrm{~V} / \mathrm{V}$ solution in Glycol Acetic Acid $2 \%$ V/V solution, mixed with Sodium Dodecyl Sulphate (SPS) $16 \%$ solution, with final pH of 4.7) was added to the mixture. Ultimately, the absorbance was measured at $570 \mathrm{~nm}^{[35]}$. Fibroblast cells and three types of cancerous cells including (MCF-7, HeLa, and HepG2) were used in this test. The cytotoxicity of compounds was computed as a percentage of controlled cells (untreated cells) and the presented values in the table are the inhibitory concentration $\left(\mathrm{IC}_{50}\right)$ of the three compounds compared to control.

\section{Docking study}

The PDB file (PDB ID: 2OYU) of crystal structure of cyclooxygenase-1 was downloaded from the RCSB Protein Data Bank. The co-crystallized ligand was removed and the receptor was prepared by Autodock Tools (ver 1.5.6rc3) ${ }^{[36]}$.The structure of ligands were sketched, minimized and saved as pdbqt format. AutoDock Vina (version 1.1.2) ${ }^{[37]}$ was used for docking study. A box with $20 \times 20 \times 20$ dimension used as search space. The centre of grid box was adjusted to be the centre of co-crystallized ligand. The results of docking were analysed by Pymol.

\section{Results And Discussion}

\section{Chemistry}

The hydrazone (1a-1j) and acylhydrazone derivatives (2a-2l) were synthesized in a single step route (Scheme 1). Phenyl hydrazine and isonicotinic acid hydrazide reacted with different aldehydes in distilled water using acetic acid as catalyst ${ }^{[24]}$. The final products obtained in satisfactory yields and purity. The 
structure of compounds was characterized by different spectroscopic techniques such as IR, ${ }^{1} \mathrm{HNMR}$ and MS.

\section{Biological evaluation}

All the synthesized compounds were evaluated for their antiplatelet activity in AA and ADP-induced aggregation of human plasma using light transmission aggregometry. The results are disclosed in Table 1 and aspirin and indomethacin were used as positive controls.

Based on the obtained results, the phenylhydrazone derivatives (1a-j) exhibited considerable activity when AA used as platelet aggregation inducer and showed no activity against ADP-induced platelet aggregation.

On the other hand the antiplatelet activity of pyridine acylhydrazone derivatives (2a-l) indicates that none of them exhibit acceptable activity against neither ADP nor AA-induced aggregation. Therefore, it can be concluded that the introduction of a carbonyl group is deleterious to the antiplatelet activity of hydrazone compounds. This has been confirmed by the previous study which reported loss of antiplatelet activity in aroylhydrazones of indole-3-carboxaldehyde ${ }^{[20]}$.

In the phenylhydrazone group, methoxyphenyl, 4-Hydroxy-3-methoxyphenyl (1c, 1g) and pyrrolesubstituted derivatives (1 $\mathbf{h})$, demonstrated acceptable antiplatelet activity against AA-induced platelet aggregation with $\mathrm{IC}_{50}$ values around 34-39 $\mu \mathrm{M}$. This finding is in agreement with previous studies [19] showing that hydrazone compounds with electron-rich heterocyclic rings could be potent inhibitors of AAinduced platelet aggregation.

Table 1 Antiplatelet activity of synthesized compounds against AA and ADP. 


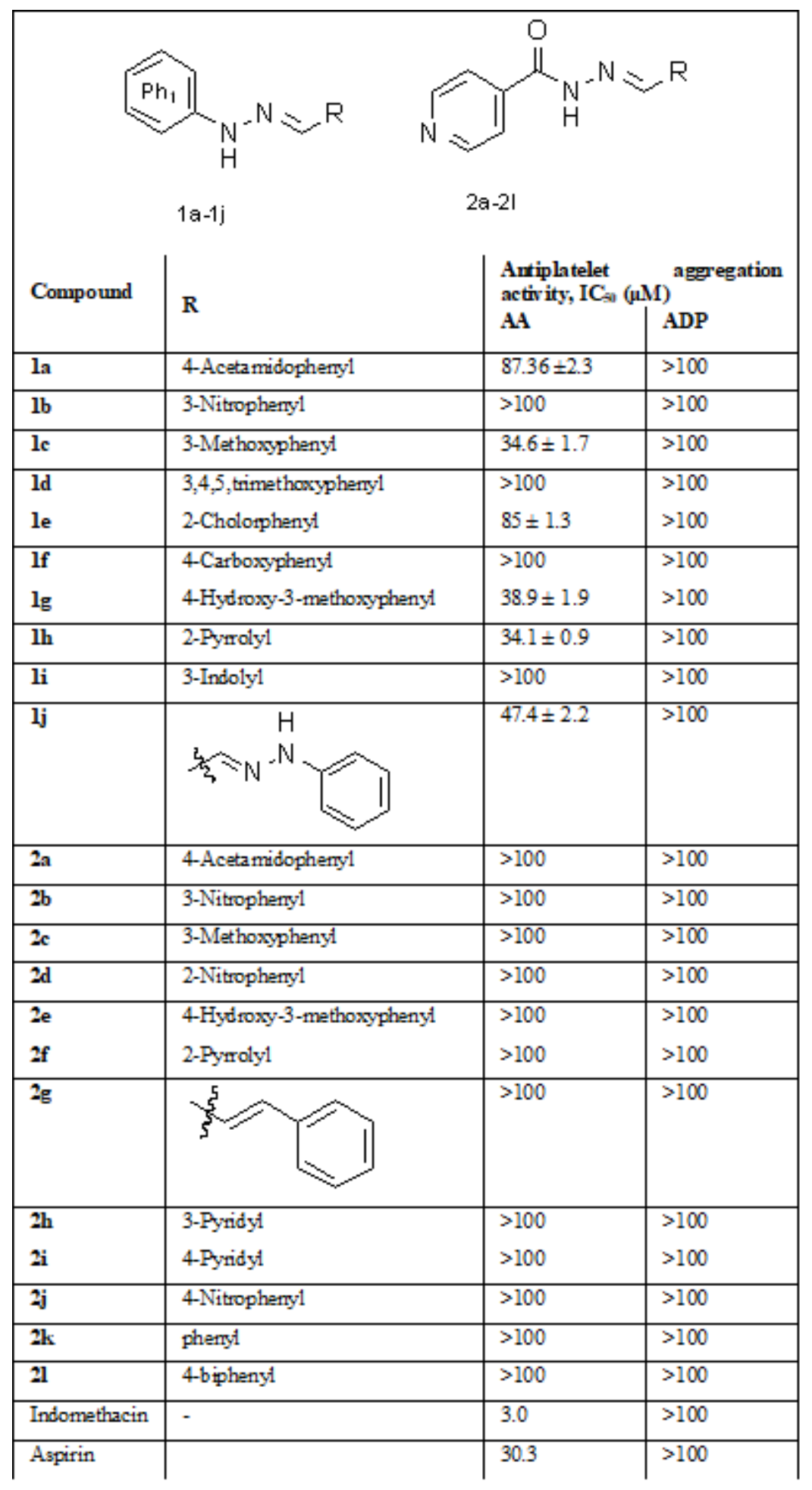

\section{Cytotoxicity assay}

Furthermore, the cell viability assessment of the most effective compounds $(\mathbf{1 c}, \mathbf{1 g}$, and $\mathbf{1 h})$ using the MTT test revealed that these compounds are fairly safe. (Table 2) Accordingly, $\mathbf{1}$ h showed no toxicity, while $1 \mathrm{c}$ and $\mathbf{1 g}$ only reached a toxic level at concentration 3 to 4 times more than their $\mathrm{IC}_{50}$ for antiplatelet activity. Moreover, $\mathbf{1 g}$ has shown toxicity on cancerous cell lines with an $\mathrm{IC}_{50}$ even less than its pharmacological effects. This observation indicates that $\mathbf{1 g}$ can be an area of research for developing an anticancer agent. 
Table 2

Cell cytotoxicity (MTT test results) of compounds with

highest antiplatelet activity in normal cell (Fibroblast) and 3

different cancer cell lines (HepG2, HeLa, and MCF-7).

\begin{tabular}{|c|c|c|c|c|}
\hline \multirow[t]{2}{*}{ Compound } & \multicolumn{4}{|c|}{ MTT assay, IC 50 ( $\mu \mathrm{M})$} \\
\hline & Fibroblast & HepG2a $^{a}$ & HeLab & MCF-7c \\
\hline $1 c$ & 214.5 & 129.6 & 220.6 & 157.6 \\
\hline $1 \mathrm{~g}$ & 163.9 & 21.00 & 17.79 & 43.17 \\
\hline $1 \mathrm{~h}$ & $>1000$ & $>1000$ & $>1000$ & $>1000$ \\
\hline Cisplatind & 9.76 & 26.96 & 9.3 & 23.89 \\
\hline \multicolumn{5}{|c|}{ a Human liver cancer cell line. } \\
\hline \multicolumn{5}{|c|}{ b Human cervical carcinoma cell line. } \\
\hline \multicolumn{5}{|c|}{ c Human breast cancer cell line. } \\
\hline \multicolumn{5}{|c|}{ d Obtained from our previous studies ${ }^{[25]}$. } \\
\hline
\end{tabular}

Docking studies

Docking studies of the most active compounds against AA-induced platelet aggregation $(\mathbf{1 c}, \mathbf{1 g}, \mathbf{1 h})$ were also achieved to check the binding interactions of compounds with the active site of cyclooxygenase 1 (COX-1) using Autodock Vina. For validation of used docking parameters, the co-crystallized ligand was docked into the active site. The obtained RMSD value (0.839) was acceptable for the validity of the docking procedure. Based on the results it was observed that hydrophobic interactions with Val349 and Gly524 are involved in the attachment of $1 \mathrm{~h}$ to the active site of COX-1. In compound $1 \mathrm{c}$ and $1 \mathrm{~g}$ in addition of hydrophobic interactions with Gly526 and Val116, hydrogen bond is also formed between Ser530 and the oxygen atom of the methoxy group of compounds1cand $\mathbf{1 g}$. The structure of the best poses of compounds $\mathbf{1 c}$ and $\mathbf{1 g}$ in the active site is shown in figure 2.

\section{Conclusion}

In this study, two groups of arylhydrazone derivatives (phenylhydrazone and Isonicotinic acid hydrazone) were synthesized and their anti-platelet activity was evaluated. Among them, arylhydrazone group demonstrates adequate activity against AA-induced aggregation with no cell toxicity at therapeutic levels. However, $\mathrm{N}$-isonicotinohydrazide derivatives were inactive in inhibiting neither ADP nor AA-induced aggregation. Developing a clear correlation between the activity and structural features requires a larger library of compounds. This is probably due to the presence of multiple receptors on the platelet surface and divergent mechanisms in charge of modulating platelet aggregation. Moreover, the studied 
compounds could have exerted the observed antiplatelet activity characteristics by interacting with multiple receptor; thus, no obvious structure activity relationship could be concluded. Additional studies are essential for clarifying the mechanism of action and developing a more potent antiplatelet agent.

\section{Declarations}

\section{Conflict of Interest}

The author declares no conflict of interest.

\section{Acknowledgements}

The authors would like to thank Kimia Tabib for her kind assistance.

\section{References}

1. Roth GA, Johnson C, Abajobir A, Abd-Allah F, Abera SF, Abyu G, Ahmed M, Aksut B, Alam T, Alam K, Alla F, Alvis-Guzman N, Amrock S, Ansari H, Ärnlöv J, Asayesh H, Atey TM, Avila-Burgos L, Awasthi A et al (2017) J Am Coll Cardiol 70:1-25

2. Mensah GA, Roth GA, Fuster V (2019) J Am Coll Cardiol 74:2529-2532

3. Okwuosa IS, Lewsey SC, Adesiyun T, Blumenthal RS, Yancy CW (2016) Int J Cardiol 202:433-440

4. Willoughby S, Holmes A, Loscalzo J (2002) Eur J Cardiovasc Nurs 1:273-288

5. Knuuti J, Wijns W, Achenbach S, Agewall S, Barbato E, Bax JJ, Capodanno D, Cuisset T, Deaton C, Dickstein K, Edvardsen T, Escaned J, Funck-Brentano C, Gersh BJ, Gilard M, Hasdai D, Hatala R, Mahfoud F, Masip J et al (2020) Eur Heart J 41:407-477

6. Capodanno D, Ferreiro JL, Angiolillo DJ (2013) J Thromb Haemost 11:316-329

7. Sostres C, Lanas A (2011) Nat Rev Gastroenterol Hepatol 8:385-394

8. Scheiman JM (2005) Rev Cardiovasc Med 6:23-31

9. Elblbesy MA, Hereba ARM, Shawki MM (2012) Int J Biomed Sci 8:188-193

10. Yeung J, Holinstat M (2012) J Blood Med 3:33-42

11. Maree AO, Fitzgerald DJ (2007) Circulation 115:2196-2207

12. Jennings LK (2009) Crit Pathways Cardiol A J Evidence-Based Med 8:20-28

13. Ben-Dor I, Kleiman NS, Lev E (2009) Am J Cardiol 104:227-233

14. Coccheri S (2010) Drugs 70:887-908

15. Rollas S, Küçükgüzel ŞG (2007) Molecules 12:1910-1939

16. De Miranda AS, Bispo W, Da Silva YKC, De Paula Castro R, Sabino JR, Lião LM, Lima LM, Barreiro E (2012) J Molecules 17:14126-14145

17. Fraga AG, Rodrigues CR, de Miranda AL, ., Barreiro EJ, Fraga CA (2000) Eur J Pharm Sci 11:285-290 
18. Silva GA, Costa LMM, Brito FCF, Miranda ALP, Barreiro EJ, Fraga CAM (2004) Bioorganic Med Chem 12:3149-3158

19. Tehrani KHME, Sardari S, Mashayekhi V, Zadeh ME, Azerang P, Kobarfard F (2013) Chem Pharm Bull 61:160-166

20. Haj ME, Tehrani K, EsfahaniZadeh M, Mashayekhi V, Hashemi M, Kobarfard F, Gharebaghi F, Mohebbi S (2015) Iran J Pharm Res 14:1077-1086

21. Cordes EH, Jencks WP (1962) J Am Chem Soc 84:832-837

22. Rao VK, Reddy SS, Krishna BS, Naidu KRM, Raju CN, Ghosh SK (2010) Green Chem Lett Rev 3:217223

23. Lourenco AL, Salvador RRS, Silva LA, Saito MS, Mello JFR, Cabral LM, Rodrigues CR, Vera MAF, Muri EMF, de Souza A, M. T., Craik CS, Dias LRS, Castro HC, Sathler PC (2017) Eur J Med Chem 135:213229

24. Mirfazli SS, Kobarfard F, Firoozpour L, Asadipour A, Esfahanizadeh M, Tabib K, Shafiee A, Foroumadi A (2014) Daru 22:65

25. Mashayekhi V, Tehrani HMohammadE, Azerang K, Sardari P, Kobarfard F (2013)Arch. Pharm. Res.113

26. Dikusar EA, Potkin VI (2009) Russ J Gen Chem 79:953-956

27. Raghuvanshi DS, Mahulikar PP, Meshram JS (2015) RSC Adv 5:48071-48078

28. Malhotra M, Sharma G, Deep A (2012) Acta Pol Pharm - Drug Res 69:637-644

29. Safoklov BB, Atovmyan EG, Nikonova LA, Tkachev VV, Aldoshin SM (2002) Russ Chem Bull 51:2224-2229

30. Habala L, Varényi S, Bilková A, Herich P, Valentová J, Kožíšek J, Devínsky F (2016) Molecules 21:1742

31. Faghih Akhlaghi M, Amidi S, Esfahanizadeh M, Daeihamed M, Kobarfard F (2014) Iran J Pharm Res 13:35-42

32. Eskandariyan Z, Esfahani Zadeh M, Tehrani HMohammadE, Mashayekhi K, Kobarfard F (2014) Arch Pharm Res 37:332-339

33. Mokhtari S, Mosaddeghb M, Moghadam MH, Soleymania Z, Ghafarib S, Kobarfard F (2012) Iran J Pharm Res 11:411-421

34. Mosmann T (1983) J Immunol Methods 65:55-63

35. Riss TL, Moravec RA, Niles AL, Duellman S, Benink HA, Worzella TJ, Minor L (2004) Cell Viability Assays. Assay Guidance Manual

36. Morris GM, Huey R, Lindstrom W, Sanner MF, Belew RK, Goodsell DS, Olson AJ (2009) J Comput Chem 30:2785-2791

37. Trott O, Olson AJ (2019) J Comput Chem 31:455-461

\section{Scheme}


Scheme 1 is available in supplementary section.

Figures

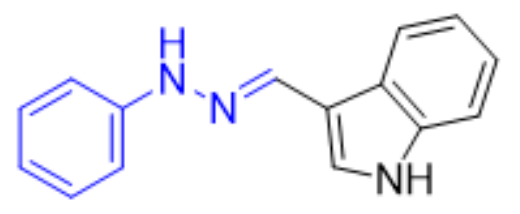

(I)

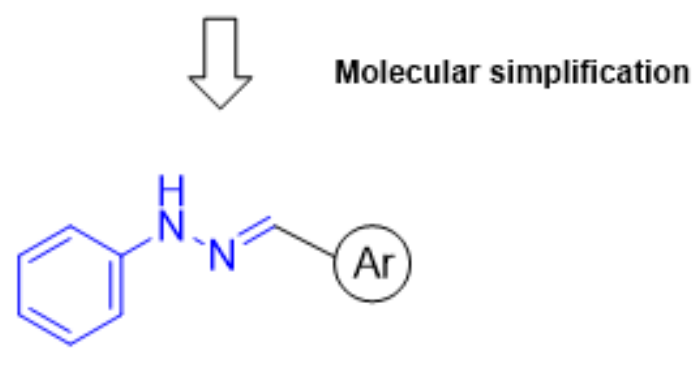

$1 a-j$
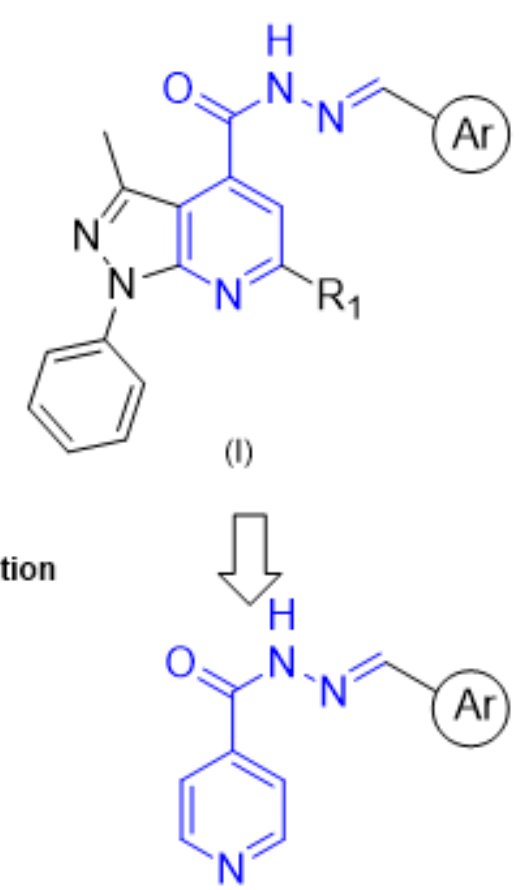

2a-I

\section{Figure 1}

General structure of designed hydrazone and N-acylhydrazone compounds. 

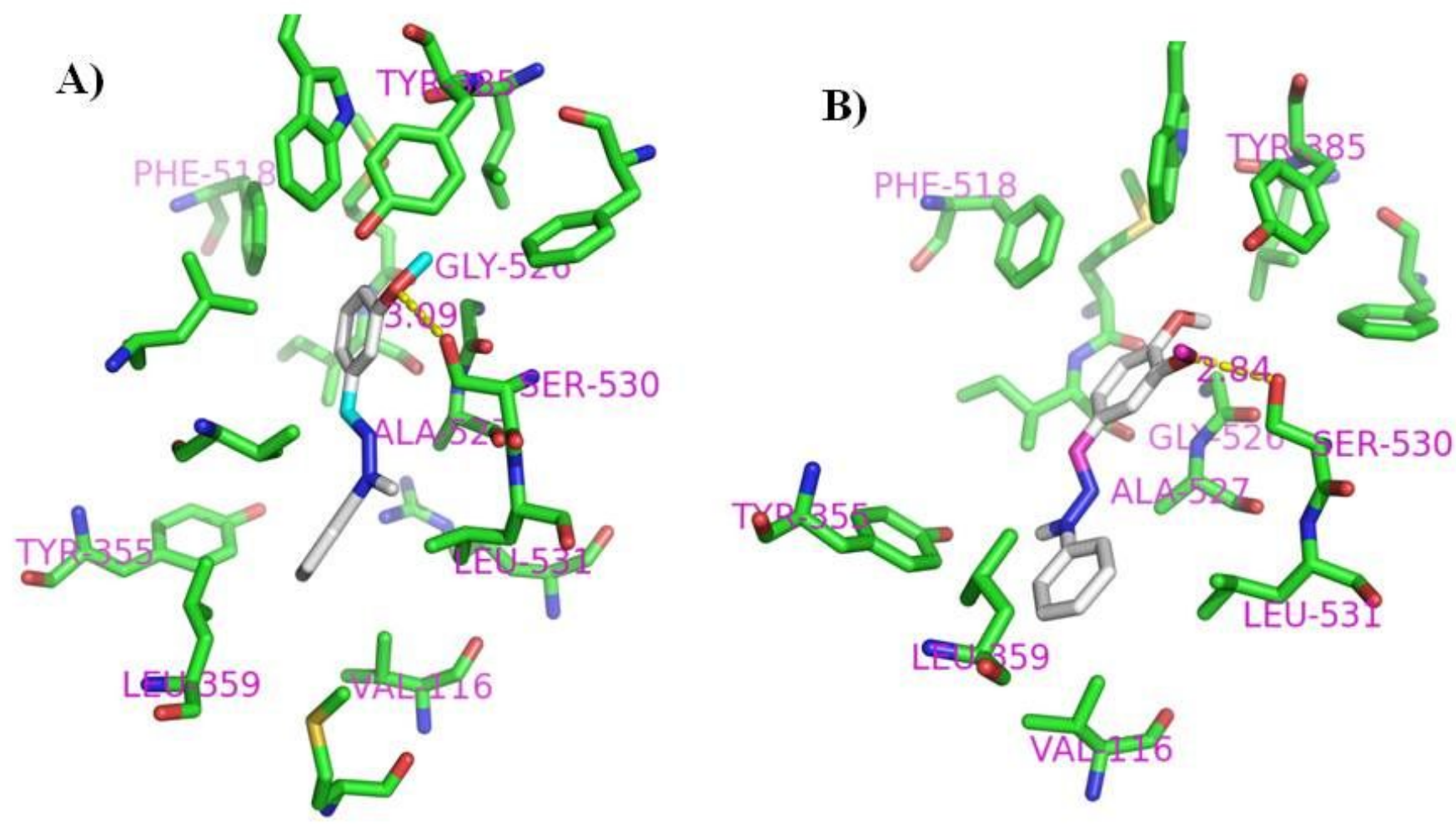

Figure 2

The binding interaction of the most active compounds $1 \mathrm{c}(\mathrm{A})$ and $1 \mathrm{~g}(\mathrm{~B})$ in the active site of COX-1. Hydrogen bond is shown by yellow dashed line.

\section{Supplementary Files}

This is a list of supplementary files associated with this preprint. Click to download.

- floatimage7.png

- Onlinefloatimage2.png

- supportingdata.docx 